\title{
Absorption enhancement of adefovir dipivoxil by incorporating MCT and ethyl oleate complex oil phase in emulsion
}

\author{
Ping LI, Hong-zhen YU, Xin-xin ZHANG, Li GAN, Chun-liu ZHU, Yong GAN* \\ Shanghai Institute of Materia Medica, Chinese Academy of Sciences, Shanghai 201203, China
}

Aim: To improve the oral absorption of adefovir dipivoxil (ADV) by employing MCT and the esterase inhibitor ethyl oleate (EO) as a complex oil phase in emulsion.

Methods: EO was used as the esterase inhibitor, and its inhibitory effect on esterase activity was assessed in rat intestinal homogenates. ADV emulsions with or without EO were prepared. The emulsions' protective effect against intestinal metabolism was evaluated in rat luminal contents, ex vivo, as well as in vivo.

Results: The $\mathrm{IC}_{50}$ of EO in intestinal mucosal homogenates was $2.2 \mathrm{mg} / \mathrm{mL}$. The emulsions exhibited significant protective effects in rat luminal contents compared to a simple suspension $(98.7 \%, 96.3 \%, 95.7 \%$ vs $74.7 \%, P<0.01)$. The permeability calculated from the emulsion containing EO was significantly different $\left(11.4 \times 10^{-6}\right.$ vs $\left.7.4 / 8.0 \times 10^{-6}, P<0.05\right)$ from the simple suspension or the emulsion without EO in an ex vivo assay. A bioavailability study in vivo revealed that emulsions containing both EO and MCT as a complex oil phase demonstrated 1.6- and 1.5-fold enhancements in area under the curve $\left(\mathrm{AUC}_{0-12}\right)$ values $(5358 \mathrm{vs} 3386 / 3618, P<0.05)$, respectively, when compared with emulsions containing EO or MCT as a single oil phase.

Conclusion: Heterotic lipid formulations (emulsions) with an esterase inhibitor (ie, EO) may be useful in protecting ester prodrugs from intestinal metabolism and increasing their oral bioavailability.

Keywords: adefovir dipivoxil; intestinal metabolism; emulsions; permeability; oral absorption

Acta Pharmacologica Sinica (2010) 31: 881-888; doi: 10.1038/aps.2010.60; published online 21 June 2010

\section{Introduction}

Ester prodrugs have been conventionally designed to enhance the membrane permeability of their parent drugs and improve the oral bioavailability ${ }^{[1-3]}$. Generally, active drugs are released once ester bonds are cleaved via either enzymatic or chemical conversion. Typically, bioactivation is mediated through enzymes such as carboxylesterase, paraoxonase and acetylcholinesterase. These enzymes are usually widely distributed throughout the body, especially in the epithelia of the intestine and liver ${ }^{[4,5]}$. Therefore, ester prodrugs are extremely prone to be hydrolyzed during oral absorption. Pre-systematic metabolism often occurs under such circumstances, leading to poor drug bioavailability.

One form of pre-systematic metabolism is intestinal firstpass metabolism, which includes luminal hydrolysis ${ }^{[6]}$ and enterocyte metabolism ${ }^{[7]}$. Luminal hydrolysis is often caused by enzymes and the $\mathrm{pH}$ of the intestinal tract. Historically, to

\footnotetext{
* To whom correspondence should be addressed.

E-mail simm2122@vip.sina.com

Received 2010-03-04 Accepted 2010-04-23
}

prevent such hydrolysis, lipid formulations were prepared. For example, in some studies ${ }^{[8,9]}$, prodrugs were incorporated into emulsions to avoid hydrolysis in the intestinal lumen to enhance the oral absorption. However, the enhancement of absorption was not as high as expected due to esterasemediated enterocyte metabolism. To further improve the absorption of prodrugs, esterase inhibitors were developed to interfere with enterocyte metabolism. In the past, various esterase inhibitors have been described, including bis $(p$ nitrophenyl) phosphate ${ }^{[10]}$, benzil or benzene sulfonamide ${ }^{[11]}$, isatins, indole-2,3-diones ${ }^{[12]}$, trifluoromethyl ketone (TFK ${ }^{[13]}$, fruit extracts ${ }^{[14,15]}$, and mixed esters ${ }^{[16]}$. However, the toxicity, pharmacological activity, and complexity of these inhibitors limited their incorporation into pharmaceutical formulations. Notably, no esterase inhibitors have been incorporated into lipid formulations to further enhance prodrug absorption. In the present study ethyl oleate (EO), one of the first compounds discovered to have esterase inhibitory effects, was incorporated into lipid formulations.

Adefovir dipivoxil (ADV), a typical ester prodrug, was able to mask the negative charges of adefovir, thereby enhancing 
its membrane permeability. However, it has poor chemical and enzymatic stability (as illustrated in scheme 1$)^{[17]} ; 32.7 \%$ was degraded after $24 \mathrm{~h}$ of incubation in $\mathrm{pH} 7.2$ buffer $^{[18]}$ and the prodrug was completely metabolized to adefovir during its transport through rat ileum ${ }^{[19]}$. Consequently, all of these chemical conversions decreased the permeability of ADV and reduced its oral bioavailability.

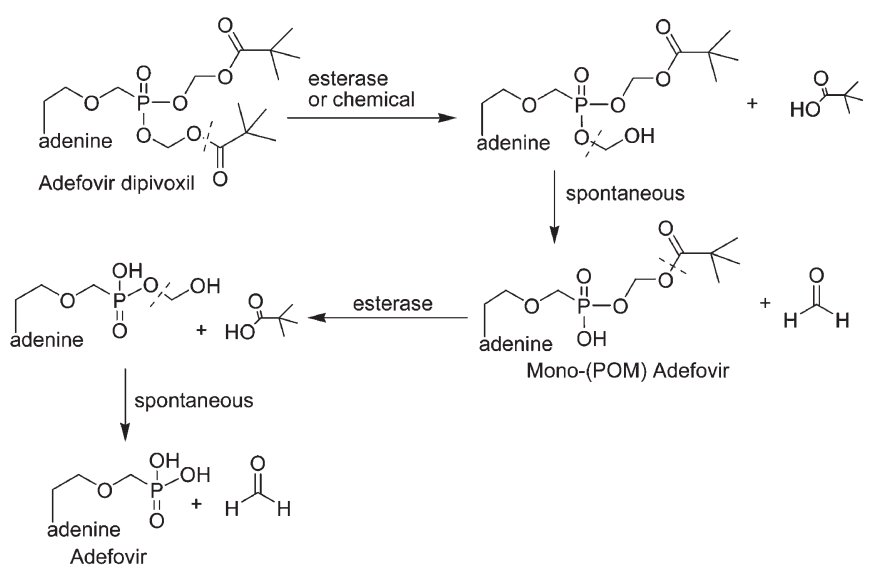

Scheme 1. Conversion of ADV in vivo.

The objective of the present study was to investigate the influence of the esterase inhibitor and the lipid formulations on the absorption of ester prodrugs. In our study, we assessed the inhibitory effect of EO on esterase activity using intestinal mucosal homogenates of rats. Furthermore, a heterotic lipid formulation containing EO (an esterase inhibitor) in the form of a complex oil phase was developed to reduce intestinal lumen hydrolysis and esterase-mediated metabolism of ADV. This lipid formulation's protective effect was verified in rat lumen, and its protective effect in enterocyte metabolism was assessed in an ex vivo experiment. We also compared its bioavailability in rats with that of reference formulations.

\section{Materials and methods}

\section{Chemicals}

Adefovir dipivoxil and adefovir (ADF) (purity >96\%) were bought from Sunheat Chemical Co (Shanghai, China). Porcine pancreatic lipase (40000 IU/g) was purchased from Kayon Biological Technology Co (Shanghai, China). Tween 80, tris (hydroxymethyl) aminomethane, maleic acid, calcium chloride, magnesium chloride, sodium chloride and EO were all from the Sinopharm Chemical Reagent Co (Shanghai, China). Triglycerides of caprylic/capric acid (MCT) were purchased from Tieling Beiya Medical Oil Co (Tieling, Liaoning, China). GMO (Peceol, containing about 46\% monoglycerides) was a gift from Gattefosse (St Priest, France). Lecithin (Epikuron 200, PC $\%=92 \%$ ) was from Lucas Meyer (Hamburg, Germany). Taurocholic sodium (NaTC) was purchased from Beijing Biolab Materials Institute (Beijing, China). Bis-p-nitrophenylphosphate (BNPP) (purity $>98 \%$ ) was purchased from Sigma
(Shanghai, China). All other chemicals were of analytical reagent grade.

\section{Animals}

Male Sprague-Dawley rats (body weight 220 20 g) were obtained from the Medical Animal Test Center of Shanghai Institute of Materia Medica (Shanghai, China). All experiments were performed according to the Shanghai Institute of Materia Medica guidelines for experimental animal care. The rats were fasted for $12 \mathrm{~h}$ prior to the experiment and had free access to water.

\section{Formulation preparation and characterization Emulsion preparation}

To study the effect of EO's on drug absorption, emulsions containing $0 \%, 6 \%$, and $12 \%$ EO were designed and termed EM1, EM2, and EM3, respectively. Emulsions were prepared according to Table 1. Preparation was generated in three steps. First, ADV was dissolved in a mixture of oils with lecithin as the oil phase; while Tween 80 dissolved in water $(\mathrm{pH}$ 6.0) was the aqueous phase. Second, oil and aqueous phases were mixed and emulsified by a high shear mixer (IKA T25, Staufen, Germany) at $12000 \mathrm{r} / \mathrm{min}$ for $5 \mathrm{~min}$ at $60^{\circ} \mathrm{C}$, thereby forming the coarse emulsion. Finally, the coarse emulsion was homogenized under 400 bar at $60^{\circ} \mathrm{C}$ with a high-pressure homogenizer (NS1001L, GEA, Sala Baganza, PR, Italy) for 8 cycles. The newly generated emulsion was saturated with nitrogen gas and kept at $4{ }^{\circ} \mathrm{C}$.

Table 1. Composition $(\%, w / w)$ of the o/w emulsions incorporating ADV*.

\begin{tabular}{lrrrrrr}
\hline Emulsion & MCT & EO & GMO & $\begin{array}{c}\text { Tween } \\
80\end{array}$ & $\begin{array}{c}\text { Epikuron } \\
200\end{array}$ & Water qs \\
\hline EM1 & 12 & 0 & 8 & 0.45 & 0.6 & 100 \\
EM2 & 6 & 6 & 8 & 0.45 & 0.6 & 100 \\
EM3 & 0 & 12 & 8 & 0.45 & 0.6 & 100 \\
\hline
\end{tabular}

${ }^{*} \mathrm{ADV}$ concentration was $2 \mathrm{mg} / \mathrm{mL}$ for each formulation.

\section{Particle size analysis}

Each sample was diluted by water (1:500) to an appropriate concentration and was determined by photon correlation spectroscopy using Nicomp380 ZLS (Particles Sizing Systems, Santa Barbara, CA, USA).

\section{Drug content and encapsulation efficiency analysis}

The total drug content in the emulsion and the encapsulation efficiency (EE) were determined by ultrafiltration-centrifugation (Microcon YM-10, 10000MW, Millipore, Bedford, MA, USA). The recovery of this method was calculated. The emulsion was centrifuged at $4000 \mathrm{r} / \mathrm{min}$ for $15 \mathrm{~min}$ and the free drug in the ultrafiltrate was determined. The EE was calculated using the following equation:

$$
\mathrm{EE}(\%)=\frac{W_{T}-W_{F}}{W_{T}} \times 100 \%
$$


where $W_{T}$ is the weight of total drug in the emulsion and $W_{F}$ is the weight of total drug in the ultrafiltrate without being encapsulated. All of the above samples were analyzed by HPLC as described below.

\section{Reference formulation preparation}

Suspensions with and without BNPP were termed Ref1 and Ref2, respectively. Suspensions were prepared as follows: ADV was ground in a mortar by gradually adding carboxymethylcellulose sodium $(0.5 \% \mathrm{w} / v)$ to a final concentration of 2.0 $\mathrm{mg} / \mathrm{mL}$. The suspension containing BNPP was also prepared as above except that BNPP was added to a final concentration of $2.8 \mathrm{mg} / \mathrm{mL}$. The particle size was measured by an optical microscope (CKX41, Olympus, Japan) and the average particle sizes $(n=100)$ were $5.8 \pm 0.5 \mu \mathrm{m}$ and $6.0 \pm 0.3 \mu \mathrm{m}$ for Ref1 and Ref2, respectively.

Evaluation of emulsions' protective effects against intestinal tract contents

\section{Preparation of intestinal tract contents}

Intestinal tract contents were prepared according to previous methods ${ }^{[6]}$. Briefly, a $20 \mathrm{~cm}$ segment of rat intestine beginning $5 \mathrm{~cm}$ distal to the pylorus was ligatured at both ends. The segment was filled with $5 \mathrm{~mL}$ of HBSS, which was incubated for $15 \mathrm{~min}$. The intestinal contents were then evacuated and spun down at $3800 \mathrm{r} / \mathrm{min}$ for $10 \mathrm{~min}$ to remove particular material. Finally the sample of intestinal tract was aliquoted and frozen at $-20^{\circ} \mathrm{C}$.

\section{ADV degradation studies with intestinal tract contents}

After optimization of incubation conditions in preliminary experiments, the protein content of the above preparations was adjusted to $0.5 \mathrm{mg} / \mathrm{mL}$ with HBSS according to the Bradford method, and bovine serum albumin (BSA) was used as the standard ${ }^{[20]}$. The enzymatic preparations $(0.5 \mathrm{mg} / \mathrm{mL})$ were pre-incubated at $37^{\circ} \mathrm{C}$ for $5 \mathrm{~min}$, and reactions were started after adding the formulations with a final drug concentration of $400 \mu \mathrm{mol} / \mathrm{L}$. The same assay conditions (without the above preparations) were established as the control group to determine $\mathrm{pH}$-mediated degradation. Every $30 \mathrm{~min}, 100$ $\mu \mathrm{L}$ of the incubation solution was collected and $500 \mu \mathrm{L}$ of icecold methanol was added to stop the reaction. Samples were vortexed for $20 \mathrm{~s}$ and centrifuged at $8000 \mathrm{r} / \mathrm{min}$ for $5 \mathrm{~min}$ at $4{ }^{\circ} \mathrm{C}$, after which the supernatant was collected and analyzed by HPLC as described below.

\section{In vitro assay for esterase inhibitory potency of EO}

\section{Preparation of intestinal mucosal homogenates}

The intestinal homogenates were prepared according to previously published methods ${ }^{[21]}$. Briefly, the rat jejunum was excised after anesthetizing the animals; subsequently, a $20 \mathrm{~cm}$ segment was cut and washed with ice-cold HBSS to remove the intestinal contents. Mucosa was removed by scraping with a glass microscope slide. The scrapings were homogenized at $0^{\circ} \mathrm{C}$ in $10 \mathrm{~mL}$ of cold HBSS for 5 min using an Ultra Turrax (IKA T10B, Staufen, Germany) at 6000 r/min. After centrifugation at $10000 \mathrm{r} / \mathrm{min}$ for $10 \mathrm{~min}$ at $4^{\circ} \mathrm{C}$, the supernatants were harvested and kept at $-20^{\circ} \mathrm{C}$. Protein content was adjusted to $0.1 \mathrm{mg} / \mathrm{mL}$ with HBSS.

\section{Inhibitory potency of EO on esterase $I C_{50}$ determination}

To determine the $\mathrm{IC}_{50}$ value (concentration of $\mathrm{EO}$ that resulted in $50 \%$ inhibition of initial esterase activity $)^{[22]}$, the enzymatic preparations $(0.1 \mathrm{mg} / \mathrm{mL})$ were preincubated for $5 \mathrm{~min}$. Then, the ADV solution [in a $5 \mathrm{mg} / \mathrm{mL}$ dimethylsulfoxide (DMSO) stock solution] together with various concentrations of EO $(0.72-5.80 \mathrm{mg} / \mathrm{mL})$ were added and incubated for $15 \mathrm{~min}$ at $37^{\circ} \mathrm{C}$. The same conditions without an inhibitor served as the control group. The final drug concentration was always 100 $\mu \mathrm{mol} / \mathrm{L}$ and the final DMSO concentration was $1 \%$, which had no effect on esterase activity ${ }^{[14]}$. The reactions were stopped by combining $100 \mu \mathrm{L}$ of the incubation solution with $200 \mu \mathrm{l}$ of icecold methanol. The samples were processed and analyzed as described in the section "ADV degradation studies with intestinal tract contents".

\section{Evaluation of emulsions' protective effect against enterocyte metabolism}

\section{In vitro dynamic lipolysis experiments}

Digestion experiments were conducted using an in vitro lipid digestion model with some modifications ${ }^{[23]}$. Briefly, $2 \mathrm{~mL}$ of ADV formulation was dispersed in $17 \mathrm{~mL}$ of digestion buffer (tris-maleate $50 \mathrm{mmol} / \mathrm{L}, \mathrm{NaCl} 150 \mathrm{mmol} / \mathrm{L}$, and $\mathrm{CaCl}_{2}$ $5 \mathrm{mmol} / \mathrm{L}, \mathrm{pH}=6.8$ ) containing $5 \mathrm{mmol} / \mathrm{L} \mathrm{NaTC}$ and 1.25 $\mathrm{mmol} / \mathrm{L}$ lecithin (conditions simulating the fasted intestinal tract). Experiments were initiated by the addition of $1 \mathrm{~mL}$ freshly made porcine pancreatic lipase solution (4000 tributyrin units $/ \mathrm{mL}$ ). The mixture was continuously stirred (100 $\mathrm{r} / \mathrm{min}$ ) and maintained at $37^{\circ} \mathrm{C}$ using a magnetic stirring apparatus. The $\mathrm{pH}$ was maintained by manual titration with $1 \mathrm{~mol} / \mathrm{L} \mathrm{NaOH}$ using a microburette with a $\mathrm{pH}$ meter (PB-10, Sartorius, Germany). The experiment was continued for 20 min and then stopped.

To achieve maximal simulation of the physiological absorption process, $7 \mathrm{~mL}$ of the post-digested mixture was immediately transferred to the subsequent permeation study ${ }^{[24]}$. Each post-digested formulation was added to the apical side as $C_{0}$ in the permeation model, and the $C_{0}$ was analyzed to determine the degradation percentage of ADV.

\section{Ex vivo studies}

Similar to previous studies ${ }^{[25]}$, the ileum portion of the intestine was excised and everted by a fine glass tube immediately after the rat was sacrificed. A sac approximately $5 \mathrm{~cm}$ in length was secured with thread at one end. A total of $1 \mathrm{~mL}$ of blank K-R solution was injected into the basal side to keep the tissue in a stretched position. After addition of $7 \mathrm{~mL}$ of the post-digestion mixture to the apical side, $200 \mu \mathrm{L}$ of basal solution was collected at 30 min intervals for $2 \mathrm{~h}$ from the basal side ( $n=3$ rats with three sacs from each rat). Afterward, the same volume of blank K-R solution was added to compensate for the volume loss. Thereafter, $400 \mu \mathrm{L}$ of ice-cold methanol 
was added to $200 \mu \mathrm{L}$ of basal solution. The samples were processed and analyzed as described in the section "ADV degradation studies with intestinal tract contents".

Apparent permeability coefficients $\left(P_{\text {app }}, \mathrm{cm} / \mathrm{s}\right)$ were calculated according to the following equation:

$$
P_{\text {app }}=\frac{d Q}{d t} \cdot \frac{1}{\mathrm{~A} \cdot C_{0}}
$$

where $d Q / d t$ is the rate $(\mu \mathrm{g} / \mathrm{s})$ of total transport of the compound and its metabolites, $A$ is the surface area $\left(\mathrm{cm}^{2}\right)$, and $C_{0}$ is the initial donor concentration $(\mu \mathrm{g} / \mathrm{mL})$.

Cellular membrane damage was evaluated by testing lactate dehydrogenase (LDH) release using the LDH kit (Nanjing Jiancheng Sheng $\mathrm{Wu}$ Yan Jiu Suo, 50T, Shanghai, China $)^{[16]}$.

\section{Pharmacokinetic studies in rats}

The fasted rats were divided into five groups with eight rats in each group ${ }^{[26]}$. All ADV formulations were administered to rats by oral gavage at a single dose of $18 \mathrm{mg} / \mathrm{kg}$ (10 mg-equivalents of $\mathrm{ADF} / \mathrm{kg})$. Blood samples $(0.5 \mathrm{~mL})$ were withdrawn by retro-orbital venous plexus puncture into heparinized tubes at 0 (predose), $0.25,0.5,1,2,4,6,8$, and $12 \mathrm{~h}$ post-dosing (four rats per time point with four or five time points per rat). All blood samples were centrifuged $(4000 \mathrm{r} / \mathrm{min}$ for $5 \mathrm{~min})$, and the obtained plasma was frozen at $-80^{\circ} \mathrm{C}$ until analysis.

\section{Analytical methods}

\section{The HPLC analysis for in vitro and ex vivo experiments}

$\mathrm{ADV}$ and its metabolites such as mono-(POM) ADF and ADF were analyzed using a previously validated HPLC method ${ }^{[7]}$. The HPLC system (Model SIL-20A, Shimadzu, Japan) was composed of an autosampler (SIL-20A), pump (LC-20AT), a diode array detector (SPD-M20A) and a working station (Shimadzu LC solution). The column used was an Intersil ODSC $_{18}$ $(4.6 \mathrm{~mm} \times 150 \mathrm{~mm}, 5 \mu \mathrm{m})$. The injected volume was $20 \mu \mathrm{L}$ and the flow rate was $1 \mathrm{~mL} / \mathrm{min}$. Mobile phase B consisted of a $50 \mathrm{mmol} / \mathrm{L}$ ammonium acetate buffer solution and $2 \mathrm{mmol} / \mathrm{L}$ tetrabutylammonium hydrogen sulfate $(\mathrm{pH} 4.0)$, while mobile phase A consisted of pure methanol. Separation was carried out with $95 \%$ of mobile phase B over a 5 min period, followed by a linear gradient from $95 \%$ to $30 \%$ of mobile phase B for 5 min and with a 5 min isocratic stage of $30 \%$ mobile phase B, which was then returned to the initial condition (95\% phase B) for 5 min. The retention time for ADV, mono-(POM) ADF and $\mathrm{ADF}$ was $17.3 \mathrm{~min}, 14.7 \mathrm{~min}$ and $5.0 \mathrm{~min}$, respectively. Concentrations of mono-(POM) ADF were determined using an ADV calibration curve. The lower limit of quantitation was $0.4 \mu \mathrm{g} / \mathrm{mL}$ for both ADV and ADF. The intra-day and interday RSD were all below $5 \%$ for ADV concentrations ranging from 0.625 to $40 \mu \mathrm{g} / \mathrm{mL}$, while the intra-day and inter-day RSD were all below $2 \%$ for ADF concentrations ranging from 0.625 to $20 \mu \mathrm{g} / \mathrm{mL}$.

\section{LC/MS/MS analysis for in vivo experiments}

The concentration of ADF in plasma was determined by a previously validated LC-MS/MS method ${ }^{[27]}$. The internal standard was 9-(3-phosphonylmethoxypropyl) adenine. The serum sample $(0.2 \mathrm{~mL})$ was pretreated by precipitating protein with methanol $(0.4 \mathrm{~mL})$, and the supernatant was injected for analysis. Analytes were separated on a Diamonsil $\mathrm{C}_{18}$ column

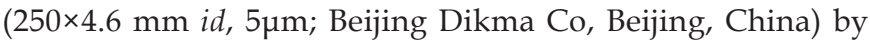
isocratic elution with a solution of methanol: water: formic acid $(20: 80: 1 \mathrm{v} / \mathrm{v} / \mathrm{v})$ at a flow rate of $0.5 \mathrm{~mL} / \mathrm{min}$, which was analyzed by an API 4000 triple-quadrupole mass spectrometric instrument (Applied Biosystems, Foster City, CA, USA) in multiple reaction monitoring mode. An electrospray ionization (ESI) source was applied and operated in the positive ion mode. Selected reaction monitoring (SRM) modes with transitions of $m / z 274 \rightarrow m / z 162$ and $m / z 288 \rightarrow m / z 176$ were used to quantify ADF and the internal standard. The linear calibration curve was obtained in the concentration range of 0.02-4.0 $\mu \mathrm{g} / \mathrm{mL}$. The lower limit of quantitation of ADF was 0.02 $\mu \mathrm{g} / \mathrm{mL}$. The inter- and intra-day precisions (RSD) were less than $5.8 \%$, and the accuracy (relative error) was within $\pm 4.5 \%$.

\section{Pharmacokinetic analysis}

Drug plasma concentrations at different time-points for individual rats were analyzed (noncompartmental analysis model) using DAS Professional software version 2.0 (Anhui, China). We calculated the peak plasma concentration of ADF $\left(C_{\max }\right)$, the time to maximum plasma concentration $\left(T_{\max }\right)$, the mean residence time between 0 and $12 \mathrm{~h}\left(\mathrm{MRT}_{0-12}\right)$, the AUC time curve between 0 and $12 \mathrm{~h}\left(\mathrm{AUC}_{0-12}\right)$ and the area under the first moment of the concentration (AUMC) time curve between 0 and $12 \mathrm{~h}\left(\mathrm{AUMC}_{0-12}\right)$. The $\mathrm{AUC}_{0-12}$ and $\mathrm{AUMC}_{0-12}$ were calculated by DAS software using the linear trapezoidal rule method. $\mathrm{MRT}_{0-12}$ was calculated as the ratio of $\mathrm{AUMC}_{0-12}$ to $\mathrm{AUC}_{0-12}$.

\section{Statistical analysis}

All values were expressed as the mean \pm standard deviation (SD). Statistical differences were determined by ANOVA followed by Tukey's test for multiple comparisons at a significance level of $P=0.01$ or 0.05 . All statistical analysis was performed using GraphPad Instat software for Windows, version 3.05 (GraphPad Software, Inc, La Jolla, CA, USA).

\section{Results \\ Emulsion characterization}

Table 2 shows the characteristics of emulsions such as particle size, polydipersity index (PI) and encapsulation efficiency (EE). The average sizes of the emulsions ranged between 240

Table 2. Particle size, polydispersity index and EE of the emulsions. Results were mean $\pm S D$ of three measures.

\begin{tabular}{cccc}
\hline Formulation & $\begin{array}{c}\text { Mean diameter } \\
(\mathrm{nm})\end{array}$ & $\mathrm{PI}$ & $\mathrm{EE}(\%)$ \\
\hline EM1 & $247 \pm 6$ & $0.104 \pm 0.017$ & $99.5 \pm 0.3$ \\
EM2 & $259 \pm 10$ & $0.089 \pm 0.043$ & $99.3 \pm 0.1$ \\
EM3 & $262 \pm 10$ & $0.050 \pm 0.022$ & $99.4 \pm 0.2$ \\
\hline
\end{tabular}


$\mathrm{nm}$ and $280 \mathrm{~nm}$. The PI was well controlled below 0.2 , and all the emulsions showed high EEs.

\section{Evaluation of emulsion's protective effects in intestinal tract contents}

The degradation of ADV when exposed to intestinal contents is shown in Figure 1. At $120 \mathrm{~min}$, the remaining percentage of ADV in the incubation mixtures of Ref1, EM1, EM2, EM3 and control were $74.7 \% \pm 1.2 \%, 98.7 \% \pm 1.5 \%, 96.3 \% \pm 1.2 \%$, $95.7 \% \pm 2.1 \%$, and $87.7 \% \pm 2.5 \%$, respectively. Results from the control group indicated the $\mathrm{pH}$-mediated degradation of ADV, while the results from the Ref1 group indicated the combined effects of $\mathrm{pH}$ and esterase. There was a significant difference between the Ref1 and the control groups for the remaining percentage of ADV at $120 \mathrm{~min}(P<0.01)$. In addition, the remaining prodrug was significantly higher in all of the emulsion groups (EM1, EM2, and EM3) compared with Ref1 and the control group at $120 \mathrm{~min}(P<0.01)$.

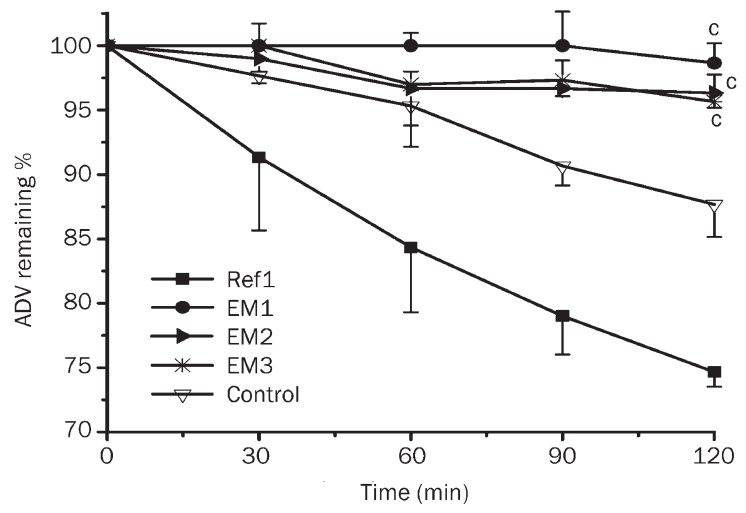

Figure 1. Time-dependent degradation of ADV in rat intestinal content, represented as the percentage of the initial prodrug concentration. Results were mean \pm SD of three determinations. ${ }^{c} P<0.01$ vs Ref 1 .

\section{Inhibitory potency of EO on esterase - $\mathbf{I C}_{50}$ determination}

To investigate the inhibitory potency of $\mathrm{EO}$ on esterase, the drug solution was incubated in the presence of various concentrations of EO in the esterase preparations. According to these results, EO inhibited the esterase activity in a concentration-dependent manner. The $\mathrm{IC}_{50}$ value was determined by plotting the relative remaining esterase activity of each test sample versus the log concentration using Origin 8.0 software. As illustrated in Figure 2, the log value of the EO concentration increased as the relative remaining esterase activity decreased. Overall, the EO was able to inhibit the esterasemediated metabolism of $\mathrm{ADV}$ in rat homogenates and the $\mathrm{IC}_{50}$ was calculated to be $2.2 \mathrm{mg} / \mathrm{mL}$.

\section{Evaluation of emulsions' protective effect on enterocyte metabolism}

The apparent permeability coefficient $\left(P_{\text {app }}\right)$ was calculated as described in the section "Ex vivo studies". $d Q / d t$ was calculated from the total drug concentration (ADV and its

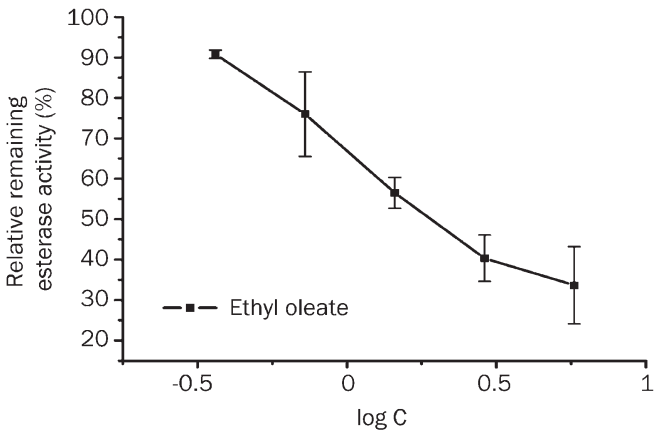

Figure 2. The $\mathrm{IC}_{50}$ determination of $\mathrm{EO}$ in intestinal homogenates. The relative remaining esterase activity was expressed as a percentage of metabolite formation compared with the control group. Results were mean $\pm S D$ of three determinations.

metabolites) at the basal side over a $30 \mathrm{~min}$ time period. The percentage of prodrug ADV (ADV\%) was calculated based on the ADV concentration and the total drug concentration $C_{0}$ at the apical side at $0 \mathrm{~min}$. As shown in Figures 3 and 4, there was no significant difference between the $P_{\text {app }}$ values for Ref1 and EM1, while the two groups were significantly different $(P<0.01)$ in regard to their $\mathrm{ADV} \%$ values. In addition, there were significant differences in $P_{\text {app }}$ values $(P<0.05)$ and ADV\% values $(P<0.01)$ between the EM2 and Ref1 samples.

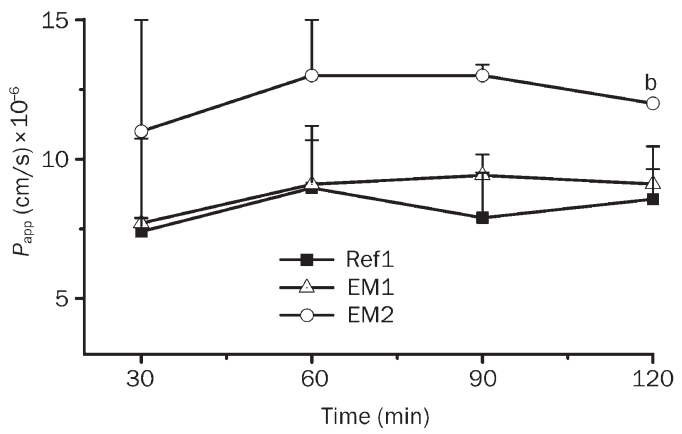

Figure 3. Apparent permeability coefficients during ex vivo experiments of rat ileum with three post-digested formulations $(400 \mu \mathrm{mol} / \mathrm{L})$. Results were mean \pm SD of three determinations. ${ }^{b} P<0.05$ vs Ref 1 and EM1.

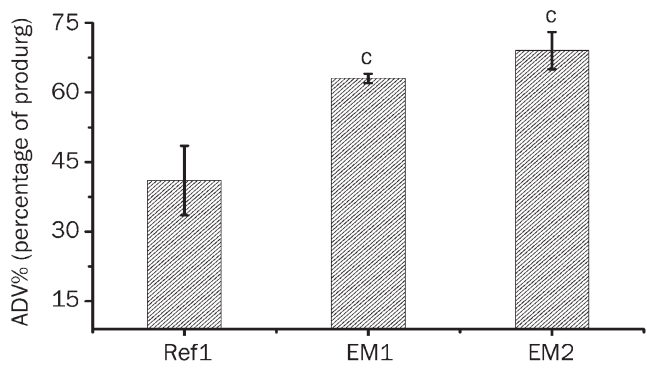

Figure 4. The prodrug ADV percentages of the $C_{0}$ after addition of three post-digested formulations to the apical side. Results were mean $\pm S D$. ${ }^{\mathrm{c}} P<0.01$ vs Ref1. 


\section{Pharmacokinetic studies}

The mean pharmacokinetic parameters derived from a noncompartmental analysis are presented in Table 3. The plasma concentration-time profiles of ADF are shown in Figure 5. The $\mathrm{AUC}_{0-12}$ of EM1 was significantly different $(P<0.05)$ from that of Ref1, which revealed the emulsion's protective effect in vivo. Notably, as the EO was introduced into the EM2 emulsion, the $\mathrm{AUC}_{0-12}$ of EM2 was significantly different $(P<0.01)$ from that of Ref1 and was also significantly different $(P<0.01)$ from that of EM1, which suggested that EM2 had an additional esterase inhibitory effect. For EM3, which contained more EO, its AUC $_{0-12}$ was significantly different $(P<0.05)$ from that of Ref1; yet, it was not significantly different from that of EM1. Meanwhile, Ref2 was significantly different $(P<0.01)$ from Ref1 in regard to $\mathrm{AUC}_{0-12}$, indicating that the esterase inhibitor BNPP may serve an important role in vivo. The $C_{\max }$ of EM2 and Ref2 was significantly different $(P<0.01)$ compared with that of Ref1 and also exhibited a significant difference with EM1 and EM3 $(P<0.05)$.

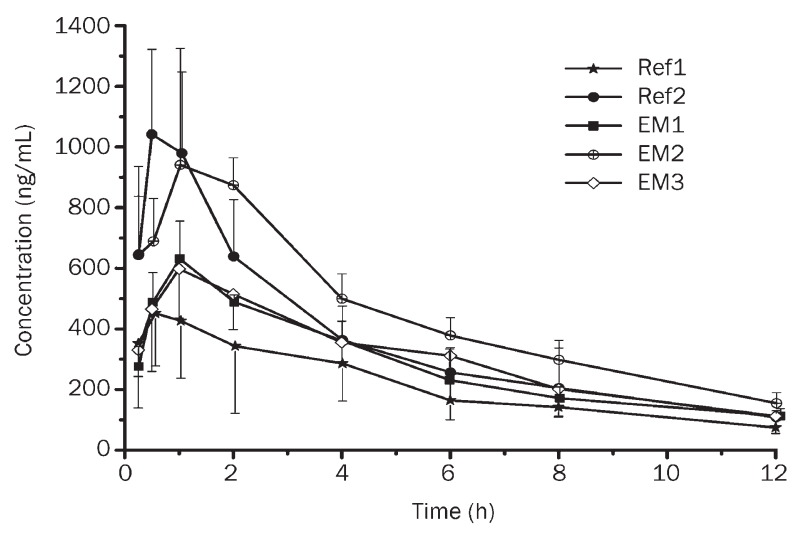

Figure 5. Adefovir plasma concentration after oral administration of different formulations. All values were mean $\pm S D$ of four determinations. $n=4$.

\section{Discussion}

\section{Selecting EO as the esterase inhibitor}

This study was designed to investigate the synergism of emulsions and esterase inhibitors on the absorption of ester prodrugs. In addition to the reported protective effects of these emulsions, the principal purpose of this study was to find a suitable esterase inhibitor. Thus, the esterase inhibitor was chosen based on the following requirements: safety, effectiveness and feasibility in oral formulations for a functional excipient.

According to the literature, long-chain alkyl esters like EO can be used as the oil phase in oral lipid formulations ${ }^{[28,29]}$. However, short-chain alkyl esters have not been reported as being utilized for the oil phase, even though they have been reported as effective esterase inhibitors. In addition, according to the literature, EO may be hydrolyzed by esterases in vivo ${ }^{[30,31]}$, which indicates that EO can inhibit esterase in a competitive manner. This hypothesis was demonstrated in our study (data not shown), and our data determined the inhibitory potency of $\mathrm{EO}$ against esterases $\left(\mathrm{IC}_{50}=2.2 \mathrm{mg} / \mathrm{mL}\right)$. The highest dose of EO used in our study was $1.0 \mathrm{~g} / \mathrm{kg}$ per rat, which was less than the safest recommended dose of $5.5 \mathrm{~g} / \mathrm{kg}$ per rat reported in the literature ${ }^{[30,32]}$. Therefore, EO was chosen as a potent and safe inhibitor of esterases and was incorporated into emulsions as the oil phase.

The protective effect of emulsions on the enhancement of oral absorption for ADV

The protective effect of the emulsions was verified by incubation with intestinal tract contents, which demonstrated that more ADV was hydrolyzed in Ref1. These results were in accordance with previous studies of other esters ${ }^{[8,9]}$. This may be due to the high encapsulation efficiency in this study, which was $99.5 \%, 99.3 \%$, and $99.4 \%$ for EM1, EM2, and EM3, respectively. Because of the encapsulation by lipids, the ester bond easily avoided long-term exposure to intestinal fluid, which would normally have caused chemical degradation and enzymatic metabolism. Furthermore, the protective effect of these emulsions was also evaluated by the combination of in vitro digestion and ex vivo permeation experiments, which closely simulated the intestinal absorption process in vivo ${ }^{[24]}$. These results showed that more prodrug was hydrolyzed in the Ref1 group in the digestion test compared to emulsions groups (Figure 4), which may be attributed to the protective effect of post-digested emulsions. During the digestion process of emulsions, micelles and vesicles are formed, thereby leading to an efficient encapsulation of ADV. Hence, only a part of the free prodrug dissociates from the digestion product and is hydrolyzed $^{[33]}$. Accordingly, there is more remaining ADV in emulsions, since the prodrug ADV is more permeable than its

Table 3. Pharmacokinetic parameters of formulations ${ }^{*}$. All values were mean \pm SD. $n=4 .{ }^{b} P<0.05,{ }^{c} P<0.01$ vs Ref1. ${ }^{e} P<0.05$ vs EM2.

\begin{tabular}{|c|c|c|c|c|c|}
\hline Parameters & Ref1 & Ref2 & EM1 & EM2 & EM3 \\
\hline $\mathrm{AUC}_{0-12}(\mathrm{mg} / \mathrm{L} * \mathrm{~h})$ & $2248 \pm 568$ & $4253 \pm 883^{c}$ & $3386 \pm 550^{\text {be }}$ & $5358 \pm 414^{\circ}$ & $3618 \pm 933^{\text {be }}$ \\
\hline $\mathrm{MRT}_{0-12}(\mathrm{~h})$ & $4.5 \pm 0.3$ & $3.8 \pm 0.6$ & $4.3 \pm 0.2$ & $4.3 \pm 0.4$ & $4.4 \pm 0.5$ \\
\hline$t_{\max }(\mathrm{h})$ & $0.6 \pm 0.2$ & $0.6 \pm 0.3$ & $1.0 \pm 0.0$ & $1.3 \pm 0.5$ & $1.0 \pm 0.0$ \\
\hline$C_{\max }(\mathrm{mg} / \mathrm{L})$ & $451 \pm 173$ & $1042 \pm 280^{\circ}$ & $631 \pm 124^{\mathrm{e}}$ & $1060 \pm 272^{c}$ & $598 \pm 161^{e}$ \\
\hline $\operatorname{Fr}(A \cup C s / A \cup C r)$ & $1.0 \pm 0.2$ & $1.9 \pm 0.4$ & $1.5 \pm 0.2$ & $2.4 \pm 0.2$ & $1.6 \pm 0.4$ \\
\hline
\end{tabular}

\footnotetext{
* “AUCs" stands forinvestigated formulation's $\mathrm{AUC}_{0-12} ;$;AUCr" stands for Ref1's $\mathrm{AUC}_{0-12}$.
} 
metabolites ${ }^{[7]}$; consequently, the emulsions' overall permeability was increased. However, the protective effect was limited in regard to membrane permeability enhancement, which may explain why there were no differences between EM1 and Ref1 in regard to $P_{\text {app }}$ values (Figure 3).

Finally, the protective effect of these emulsions was also evaluated in vivo. EM1 contained MCT as the oil phase, which had no inhibitory effect on esterase activity but good protective effects against degradation. Therefore, this emulsion only reduced pre-absorption hydrolysis to some extent and showed limited enhancing effects on absorption. The $\mathrm{AUC}_{0-12}$ of $\mathrm{ADV}$ administrated as an EM1 emulsion was 1.5-fold higher than the value of Ref1.

The esterase inhibitory effect of EO on oral absorption enhancement of ADV

BNPP is a well-known esterase inhibitor ${ }^{[10,34]}$ and was demonstrated to enhance the stability of ester prodrugs in the lumen and enterocytes, thereby resulting in better oral absorption. Thus, the $\mathrm{AUC}_{0-12}$ of Ref2 was 1.9-fold higher in rats compared with that of Ref1, indicating that esterase inhibitors play an important role in the oral absorption of ADV. Since EO is also an effective esterase inhibitor, it was expected to interfere with the esterase-mediated metabolism of ADV and to improve its overall oral absorption. Therefore, EM3 (which contained EO as the oil excipient) improved the oral bioavailability by 0.6 fold compared with Ref1.

In addition, due to the esterase inhibitory effect of EO, EM2 showed enhanced membrane permeability compared with EM1 in the ex vivo study and the $\mathrm{AUC}_{0-12}$ of EM2 was 1.6-fold higher compared to EM1.

\section{The heterotic lipid formulation may serve as a better carrier for ester prodrugs}

Both EM1 and EM3 enhanced the oral absorption of ADV, but the enhancement was not as high as Ref2. However, EM2 displayed an even greater enhancement in oral absorption than that of Ref2, although EO $\left(\mathrm{IC}_{50}=2.2 \mathrm{mg} / \mathrm{mL}\right)$ showed a worse inhibitory potency than $\mathrm{BNPP}\left(\mathrm{IC}_{50}=0.1 \mu \mathrm{g} / \mathrm{mL}\right)$. Moreover, in spite of the fact that EM3 contained more $\mathrm{EO}$, the $\mathrm{AUC}_{0-12}$ of EM2 was 1.5 fold-higher than that of EM3.

Generally, triglycerides are used in lipid formulations as the oil excipient to enhance the oral bioavailability of poorly water-soluble drugs. In contrast, ethyl esters of fatty acids have been rarely used ${ }^{[35]}$. This differential utilization may be due to different in vivo behaviors of these excipients after oral absorption. Triglycerides are mainly hydrolyzed in the lumen by lipase-colipases into monoglycerides and fatty acids, which rapidly form into mixed micelles and vesicles with bile salts and phospholipids in vivo ${ }^{[36]}$. These digested colloids efficiently encapsulate the hydrophobic drug in intestine, thereby increasing solubilization and stability of the drug ${ }^{[33,37]}$. However, the esters of fatty acids such as ethyl esters are quickly digested into fatty acids and ethanol in the intestinal tract ${ }^{[30]}$. Subsequently, these digested products are then formed into micelles with relative poor encapsulation capacity ${ }^{[37]}$, resulting in a relatively poor enhancing effect in bioavailability.

In the present study, emulsions were designed with different ratios of MCT to EO as oil phases. This heterotic lipiddelivery system EM2, which contained a 1:1 ratio of (MCT:EO) as the oil phase, showing the greatest enhancement of bioavailability among all of the emulsions. Notably, the EM2 provided both physical protection of the prodrug and an esterase inhibitory effect in vivo. On the one hand, compared with EM3, the highly loaded MCT in EM2 could be digested into vesicles, which provide good physical protection of ADV and $\mathrm{EO}$ in the lumen ${ }^{[37]}$. However, EO in the EM3 was rapidly digested into fatty acids and ethanol in the intestine, which may have accelerated the release of ADV from the EM3. ADV may have been exposed to hydrolysis in the intestinal fluid, reducing absorption-enhancing effects. On the other hand, compared with EM1, the EM2 had an additional esterase inhibitory effect so EM2 could reduce the esterase-mediated metabolism during absorption, thus increasing the absorptionenhancing effect.

In conclusion, our results showed that $\mathrm{EO}$ was an effective inhibitor of esterases. In addition, the heterotic lipid formulation that contained both EO and MCT as the oil phase exhibited a higher bioavailability of ADV in comparison to Ref1, EM1, and EM3 formulations. These effects were possibly due to a reduction of intestinal first-pass metabolism via the protective effects of this emulsion and EO's inhibitory effect on esterases. Furthermore, bioavailability results suggested that this novel heterotic emulsion might be a potentially useful formulation for the enhanced bioavailability of ester prodrugs.

\section{Acknowledgements}

This work was supported by the National Natural Sciences Foundation of China (No 30701054) and the National Science \& Technology Major Project's Key New Drug Creation and Manufacturing Program (№ 2009ZX09301-001). This work was also supported in part by the National Basic Research Program of China (No 2009CB930300).

\section{Author contribution}

Yong GAN designed the research; Ping LI performed the research and wrote the paper; Xin-xin ZHANG and Chunliu ZHU contributed to the development of new analytical reagents and tools; Li GAN and Hong-zhen YU analyzed the data.

\section{References}

1 Beaumont K, Webster R, Gardner I, Dack K. Design of ester prodrugs to enhance oral absorption of poorly permeable compounds: Challenges to the discovery scientist. Curr Drug Metab 2003; 4: 461-85.

2 Ettmayer P, Amidon GL, Clement B, Testa B. Lessons learned from marketed and investigational prodrugs. J Med Chem 2004; 47: 2393-404.

3 Testa B. Prodrug research: Futile or fertile? Biochem Pharmacol 2004; 68: 2097-106.

4 Bianca ML, Ronald TB. Enzymes Involved in the bioconversion of ester-based prodrugs. J Pharm Sci 2006; 95: 1177-95. 
5 Imai T. Human carboxylesterase isozymes: Catalytic properties and rational drug design. Drug Metab Pharmacokinet 2006; 21: 173-85.

6 Crauste-Manciet S, Huneau JF, Decroix MO, Farinotti R, Chaumeil JC. Cefpodoxime proxetil esterase activity in rabbit small intestine: a role in the partial cefpodoxime absorption. Int J Pharm 1997; 149: 241-9.

7 Annaert P, Kinget R, Naesens L, De Clercq E, Augustijns P. Transport, uptake, and metabolism of the bis(pivaloyloxymethyl)-ester prodrug of 9-(2-phosphonylmethoxyethyl)adenine in an in vitro cell culture system of the intestinal mucosa (Caco-2). Pharm Res 1997; 14: 492-6.

8 Crauste-Manciet S, Brossard D, Decroix MO, Farinotti R, Chaumeil JC. Cefpodoxime proxetil protection from intestine lumen hydrolysis by oilin water submicron emulsions. Int J Pharm 1998; 165: 97-106.

9 Cao F, Guo JX, Ping QN, Liao ZG. Prodrugs of scutellarin: ethyl, benzyl and $\mathrm{N}, \mathrm{N}$-diethylglycolamide ester synthesis, physicochemical properties, intestinal metabolism and oral bioavailability in the rats. Eur J Pharm Sci 2006; 29: 385-93.

10 Brandt E, Heymann E, Mentlein R. Selective inhibition of rat liver carboxylesterases by various organophosphorus diesters in vivo and in vitro. Biochem Pharmacol 1980; 29: 1927-31.

11 Hyatt JL, Tsurkan L, Wierdl M, Edwards CC, Danks MK and Potter PM. Intracellular inhibition of carboxylesterases by benzil: modulation of CPT-11 cytotoxicity. Mol Cancer Ther 2006; 5: 2281-8.

12 Hyatt JL, Moak T, Hatfield MJ, Tsurkan L, Edwards CC, Wierdl M, et al. Selective Inhibition of carboxylesterases by isatins, indole-2,3-diones. J Med Chem 2007; 50: 1876-85.

13 Harada T, Nakagawa Y, Wadkins RM, Potter PM, Wheelock CE. Comparison of benzil and trifluoromethyl ketone (TFK)-mediated carboxylesterase inhibition using classical and 3D-quantitative structure-activity relationship analysis. Bio Org Med Chem 2009; 17 : 149-64.

14 Van Gelder J, Annaert P, Naesens L, De Clercq E, Mooter GV, Kinget R, et al. Inhibition of Intestinal metabolism of the antiviral ester prodrug bis(poc)-PMEA by nature identical fruit extracts as a strategy to enhance its oral absorption: an in vitro study. Pharm Res 1999; 16: 1035-40.

15 Augustijns P. Increased absorption of the antiviral ester prodrug tenofovir disoproxil in rat ileum by inhibiting its intestinal metabolism. Drug Metab Dispos 2000; 28: 1394-6.

16 Van Gelder J, Deferme S, Naesens L, De Clercq E, Mooter GV, Kinget $\mathrm{R}$, et al. Intestinal absorption enhancement of the ester prodrug tenofovir disoproxil fumarate through modulation of the biochemical barrier by defined ester mixtures. Drug Metab Dispos 2002; 30 : 924-30.

17 Maria F, Raza O. Case study: Adefovir dipivoxil: An oral prodrug of Adefovir. In: Biotechnology: Pharmaceutical Aspects v 5. Prodrugs: Challenges and Rewards. New York: Springer New York; 2008. p 1151-61.

18 Samira B, Helene P, Richard J, Georges M, Jean-Louis I, Anne-Marie A, et al. Synthesis, in vitro antiviral evaluation, and stability studies of bis(S-acyl-2-thioethyl) ester derivatives of 9-[2-(phosphonomethoxy) ethyl]adenine (PMEA) as potential PMEA prodrugs with improved oral bioavailability. J Med Chem 1996; 39: 4958-65.

19 Annaert P, Tukker JJ, Van Gelder J, Naesens L, De Clercq E, Van den Mooter G, et al. In vitro, ex vivo, and in situ intestinal absorption characteristics of the antiviral ester prodrug adefovir dipivoxil. J Pharm Sci 2000; 89: 1054-62.

20 Bradford MM. A rapid and sensitive method for the quantitation of microgram quantities of protein utilizing the principle of protein-dye binding. Anal Biochem 1976; 72: 248-54.

21 Van Gelder J, Shafiee M, De Clercq E, Penninckx F, Mooter GV, Kinget $\mathrm{R}$, et al. Species-dependent and site-specific intestinal metabolism of ester prodrugs. Int J Pharm 2000; 205: 93-100.

22 Ren X, Mao X, Cao L, Xue K, Si L, Qiu J, et al. Nonionic surfactants are strong inhibitors of cytochrome P4503A biotransformation activity in vitro and in vivo. Eur J Pharm Sci 2009; 36: 401-11.

23 Dahan A, Hoffman A. Use of a dynamic in vitro lipolysis model to rationalize oral formulation development for poor water soluble drugs: Correlation with in vivo data and the relationship to intra-enterocyte processes in rats. Pharm Res 2006; 23: 2165-74.

24 Dahan A, Hoffman A. The effect of different lipid based formulations on the oral absorption of lipophilic drugs: the ability of in vitro lipolysis and consecutive ex vivo intestinal permeability data to predict in vivo bioavailability in rats. Eur J Pharm Biopharm 2007; 67: 96-105.

25 Parsons DS, Paterson GR. Movement of fluid and glucose in an everted sac preparation of rat colonic mucosa. Biochim Biophys Acta 1960; 41: 173-5.

26 Jeng-Pyng S, Michael SL, Krishnamurthy VV, Murty NA, Robert JJ, Alison MB, et al. Pharmacokinetics and metabolism of selected prodrugs of PMEA in rats. Drug Metab Dispos 1997; 25: 362-6.

27 Zhao LY, Chen XY, Zhang Y, Yang HY, Zhong DF. Determination of adefovir in monkey plasma by liquid chromatography-tandem mass spectrometry. Acta Pharm Sin 2003; 38: 120-3.

28 Sugiura S, Nakajima M, Yamamoto K, Iwamoto S, Oda T, Satake M, et al. Preparation characteristics of water-in-oil-in-water multiple emulsions using microchannel emulsification. J colloid interface sci 2004; 270: 221-8.

29 Yi T, Wan J, Xu H, Yang X. A new solid self-microemulsifying formulation prepared by spray-drying to improve the oral bioavailability of poorly water soluble drugs. Eur J Pharm Biopharm 2008; 70: 439-44.

30 Michael L. Fatty acid ethyl esters: nonoxidative ethanol metabolites with emerging biological and clinical significance. Lipids 1999; 34: 281-5.

31 Kaphalia BS, Fritz RR, Ansari GAS. Purification and characterization of rat liver microsomal fatty acid ethyl and 2-chloroethyl ester synthase and their relationship with carboxylesterase. Chem Res Toxicol 1997; 10: 211-8.

32 Saghir M, Werner J, Laposata M. Rapid in vivo hydrolysis of fatty acid ethyl esters, toxic nonoxidative ethanol metabolites. Am J Physiol 1997; 273: G184-90.

33 Porter CJH, Trevaskis NL, Charman WN. Lipids and lipid-based formulations: optimizing the oral delivery of lipophilic drugs. Nat Rev Drug Discov 2007; 6: 231-48.

34 Kenji M, Mitsuru H, Teruko I. Intestinal first-pass metabolism via carboxylesterase in rat jejunum and ileum. Drug Metab Dispos 2007; 35: 1089-95.

35 Porter $\mathrm{CJH}$, Pouton CW. Formulation of lipid-based delivery systems for oral administration: materials, methods and strategies. Adv Drug Delivery Rev 2008; 60: 625-37.

36 Embleton JK, Pouton CW. Structure and function of gastro-intestinal lipases. Adv Drug Delivery Rev 1997; 25(1): 15-32.

37 Porter CJH, Kaukonen AM, Taillardat-Bertschinger A, Boyd BJ, O'Connor $\mathrm{JM}$, Edwards GA, et al. Use of in vitro lipid digestion data to explain the in vivo performance of triglyceride-based oral lipid formulations of poorly water-soluble drugs: studies with halofantrine. J Pharm Sci 2004; 93: 1110-21. 